\title{
THE TEACHING OF ASTRONOMY AT THE UNITÀ DI RICERCA ASIAGO
}

\author{
Laura Erculiani Abati
}

Unità di Ricerca Asiago, c/o Osservatorio Astronomico, Vicolo dell'Osservatorio 5, 35122 Padova, Italy

The Unità di Ricerca (U.d.R.) Asiago, of the National Astronomical Group of the Italian Research Council, consists of:

1) the staff of the Padua-Asiago Observatory

2) the staff of the Astronomy Department of Padua University

3) external members with interests in stellar, galactic, and extragalactic astronomy and in technology.

The teaching of astronomy at the U.d.R. Asiago has several aspects: popularization, educational visits, cooperation with amateur groups, promotional contacts with public institutions, participation in a commission on school programs, and participation in the educational activities of the Italian Astronomical Society.

\section{Popularization}

Almost all the members of the U.d.R. give public lectures and seminars in the schools, with the aim of providing a complete view of astronomy from its historical evolution to the more recent scientific discoveries with an emphasis on acquainting people with the latest research.

\section{Educational Visits}

Many schools and private groups ask to visit the Astronomical Observatory of Asiago, but, in spite of the staff's trying, not all requests can be satisfied. There is a standard program for the visit which, besides explaining the instruments in the dome, includes slides of celestial objects and describing the main fields of astronomical research and the future of astronomy. However, the program is flexible, according to the visitors' specific requests. These visits take a large amount of staff time, but it seems to us that lectures, however useful, cannot substitute for the direct contact with the instruments and with research.

\section{Cooperation with Amateur Astronomers' Associations}

In our region (the north-east of Italy) numerous amateur astronomers' groups, some of them extremely active, exist and interact profitably with the U.d.R. In the field of research, professional astronomers offer advice, suggesting programs for different instruments and different observers, provide materials at the larger telescopes, and write papers for amateur and professional newspapers. In the field of popularization, amateur astronomers are irreplaceable; for example, they guide 
the sky observations with the naked eye and with small instruments, organize local or traveling astronomical exhibitions, manage planetariums, and conceive new experiences particularly suitable for understanding celestial phenomena.

\section{Promotional Contacts with Public Institutions}

Some members of the U.d.R. cooperate with public institutions that are devoted to educating the population and training teachers. They propose astronomical subjects and give lessons, seminars, and courses. They act as reference points both for the institutions and for people who like to get deeper knowledge of specific subjects. It seems to us that this kind of cooperation is particularly important because it places the culture of the professional astronomers at citizens' and teachers' disposal.

\section{Participation in School Programs Commission}

A member of the U.d.R. belongs to the joint commission of the Italian Astronomical Society (S.A.It.) and the Physics Teaching Association; the commission's aim is to set the astronomical teaching program of the high school. So far there are new programs for elementary and the middle secondary school, but the programs for high school are old and generic. Furthermore, only a few of the teachers ever attended a regular astronomy course during their university studies. Thus astronomy teaching in the high schools is often superficial and inadequate. We hope to help in formulating suitable and specific programs, especially in view of the high school reform that has been long expected and is probably near at hand. We believe that setting an adequate program is important, since teacher training and textbooks are planned on the basis of the Ministry's programs.

\section{Participation in the Educational Activities of the S.A.It.}

The Italian Astronomical Society, through its educational commission, promotes initiatives for the teaching of astronomy at all levels. A member of the U.d.R. belongs to this Commission and many others are involved in the different activities. A particularly significant activity is the National Summer School for teachers; it has existed for three years in collaboration with teachers' associations and is supported by the Ministry of Public Education. It is a kind of residential school for small groups of teachers; it trains them in a specific astronomical subject, either in a field of science or on methodology; it includes lessons, practical exercises, and night observations. The U.d.R. helps in all phases: proposing the arguments, giving lectures, making practical exercises, coordinating activities during the school, and writing the final proceedings. U.d.R.'s work is important for improving teaching.

In conclusion: at the U.d.R. Asiago, we are trying to develop the teaching of astronomy in all its aspects. We believe that research, education, and popularization are different ways of promoting astronomy and that promoting astronomy helps humanity. 